\title{
EEG signal analysis and characterization for the aid of disabled people
}

\author{
M. B. I. Reaz ${ }^{1}$, M. S. Hussain ${ }^{1}$, M. I. Ibrahimy ${ }^{1} \&$ F. Mohd-Yasin ${ }^{2}$ \\ ${ }^{I}$ Department of Electrical and Computer Engineering, \\ International Islamic University Malaysia, Malaysia \\ ${ }^{2}$ Faculty of Engineering, Multimedia University, Malaysia
}

\begin{abstract}
The effectiveness of assistive devices for disabled people is often limited by the human machine interface. This research proposes an intelligent wheelchair system especially for severely disabled people based on analysing electroencephalographic signals by using discrete wavelet transform and higher order statistical methods. The system to be implemented in Field Programmable Gate Array enables an accurate and efficient system of processing signals to control the wheelchair, which makes an attractive option in the hardware realization.
\end{abstract}

Keywords: EEG, wavelet, higher order statistic, bispectrum, FPGA, wheelchair.

\section{Introduction}

As a result of accident or disease, millions of people worldwide suffer from loss of motor function. These people are forced to accept a reduced quality of life dependent on other individuals. Many assistive devices targeted at the integration of disabled individuals into society have been developed in the past, but the effectiveness of these aids for individuals with severe disabilities is often limited by the human machine interface. Probably, the most important one, as far as people with physical disabilities who are unable to walk are concerned, is a wheelchair.

Nakanishi et al. [1] in 1999 proposed a powered wheelchair controlled by the face directional gestures recognized by a high-speed image processing hardware. However they did not discriminate whether the given gesture is intentional or unintentional behaviour. A Hands-free wheelchair control system relied on muscle contraction was proposed by Felzer and Freisleben [2] in 2002. 
According to the wheelchair, a constant electromyography (EMG) signal associated with any arbitrary muscle of the wheelchair driver is monitored and reduced to stream of contraction events. The reduced stream affects an internal program state, which is translated into appropriate commands understood by the wheel chair electronics. The system enables the driver to navigate the chair without the need to use hands, which is useful for people who cannot use their hands temporarily or permanently. But this would not be efficient and effective for people who are suffering from severe paralysis, as it requires contraction from muscle. An intelligent robot wheelchair based on EMG, face direction gesture, and voice was also proposed by Moon et al. [3] in 2003. The user's intention is transferred to the wheelchair via the human computer interface (HCI) and then the wheelchair is controlled to the intended direction. But the system still depends on the individual's muscle contraction, gesture and voice, which cannot be useful for severe disabilities.

The concept of brain computer interface (BCI) has emerged over the last two decades of research as a promising alternative. An advance communication interface can be developed that can allow an individual to control a wheelchair. This type of interface would increase an individual's independence, leading to an improved quality of life. Most of these techniques have focused on electroencephalographic (EEG) data, which is a good basis for such communication and control channels [4]. In a typical BCI based on spontaneous EEG, the operator generates a "control signal" by continuously changing his cognitive state when he or she wants to control the device (wheelchair). The change on the cognitive state is measured as specific temporal patterns [5]. If several mental states can be reliably distinguished by recognizing parameters in EEG, then a paralysed person could communicate to a wheelchair by composing sequence of these mental stages just by imagination.

EEG recordings and the electrical activities collected on the surface of scalp can explain the physiological activities of the brain. Since the non-stationary of the EEG records, it becomes necessary to track the transient changes of the EEG signals to have a further understanding of the different brain functions and their information processing. Over the past three decades, a great deal of modern techniques, such as, computerized tomography (CT), magnetic resonance imaging (MRI) etc. continuously came into use. But EEG signal, as a nondestructive testing method, is still playing a key role in the analysis and diagnosis of the brain and investigation of brain states in different functional states [6].

Extracting the EEG in an environment where the signal-to-noise ration (SNR) can be as low as $10 \mathrm{~dB}$ requires ensuring that the artifacts caused by sources internal to the human body like Electrooclargram (EOG) and neck muscle movements are detected and filtered out accurately. The cornea-retinal potential (CRP) developed as a result of the movement of the eyeballs also causes 'blanking' of the EEG Signal due to the spikes that occur. This along with the blink related artifacts are often dominant over other electrophysiological contaminating signals like ECG and movement of body muscles. The existing de-noising techniques that are based on frequency selective filtering lead to a 
substantial loss of EEG data. Prohibiting the subjects from blinking or moving their eyeballs is not a plausible solution and in-fact the effort of the subject is ensuring that the subject does not perform the aforementioned actions can have a significant impact on the recorded EEG. Due to these factors, frequency selective filtering methods for removal of ocular artifacts from EEG recording have been and continue to be a major challenge today [7, 8]. Wavelet based denoising is an attractive alternative owing to its ability to study the time-frequency maps simultaneously.

A great deal of signal processing techniques has been widely used to study various EEG signals in practice after Hans Berger discovered the first EEG record in 1929. Among many methods for signal processing, the spectral analysis based on Fourier analysis has become a common way for the standard quantitative analysis of the energy distribution of EEG signals. Shuren and Zhong [9] presented a multi-resolution time frequency analysis for EEG signal analysis using short time Fourier transform (STFT). While, Yamaguchi [10] used Fourier analysis along with wavelet analysis to describe EEG signal. As EEG is a non-stationary signal, Fourier transform cannot describe it fully. So there is definitely a need for transform which are not only localized in frequency domain but also in time domain. The Wavelet Transform (WT) solves both of these problems by replacing modulation with scaling of achieved localized frequency. The WT has 3 features: (a) Multiresolution; (b) Constant relative bandwidth, that is, the time-widths of the wavelets is adapted to their frequency; (c) Ability to indicate signal which is localized in the time domain or frequency domain. It is called a mathematical microscope for analysing signals [11, 12].

The conventional techniques of signal processing are generally based on the first and second order moments and cumulants and their spectral (frequency domain representation) analysis. However, these techniques do not provide the full information available from the signal. Traditional system reconstruction algorithms have various limitations and considerable computational complexity and may show high variance. Higher order statistical (HOS) method is considered as an efficient tool for analysing the EEG signal due to the unique properties of HOS applied to random time series. The bispectrum or third order spectrum has the advantage of suppressing Gaussian noise. Moreover, it carries both the magnitude and phase information, which can be used to recover the system impulse function and input impulse sequence from the linear timeinvariant (LTI) system output signal. Muthuswamy et al. [13] analysed the burst patterns of EEG signal using HOS in their research work. They studied the power spectral parameters and bispectral parameters of EEG at baseline, during early recovery from an asphyxia arrest (EEG burst patterns) and during late recovery after EEG evolve into a more continuous activity. Pomfrett and Pearson suggested an EEG monitoring system, which used bispectral analysis [14]. However, all these models used the EEG signal directly without using any denoising techniques. EEG signals are frequently affected by a number of noises such as eye blinking, muscle movements etc. Thus, it is required to apply a denoising technique such as wavelet technique before further analysing the EEG signal. 
The generated EEG signal (control signal) after denoising and signal processing will show continuous changes in his cognitive state when the subject wants to control the device (wheelchair). The change on the cognitive state will be measured as specific temporal patterns. When several mental states will be distinguished by recognizing parameters in EEG, then a paralysed person will be able to communicate to a wheelchair by composing sequence of these mental stages just by imagination.

So far there is no EEG signal analysis techniques has been modelled for hardware implementation. However, an efficient hardware based EEG signalanalysing tool can work as an important instrument for an intelligent wheelchair. Field Programmable Gate Array (FPGA) is a good choice for hardware realization of such system for signal analysis and characterization. FPGA, besides offering a potential alternative to speed up the hardware realization, comes with the merits of lower cost, higher density, and shorter design cycle [15]. FPGA offers a low risk, quick time to market solution. Dynamic programming algorithms can be programmed into an FPGA. The use of FPGA allows the hardware to be changed as easily as changing software. The FPGA implementation as a hardware prototype will enable an accurate and efficient hardware implementation of the processing of EEG signal (control signal) to control the wheelchair.

\section{Proposed design methodology}

EEG signal could be captured using EEG sensors/electrodes. EEG signal characteristics will be observed in 1-4 Hz frequency band, so an amplifier could be designed to intensify the signal for further filtering and signal processing. The EEG signals will be denoised (noise removal technique) using discrete wavelet transform (DWT) and threshold methods. Bispectrum analysis, a form of HOS, will be performed for the analysis and characterization of the EEG signal. Software simulations will be conducted with Matlab. For hardware implementation, the system will be further modelled using very high-speed integrated circuit hardware description language (VHDL). The system will then be simulated and synthesized for FPGA implementation. Figure 1 shows the flow of the algorithm. For the proposed system, left, right and forward movement will be considered where mental states will be reliably distinguished by recognizing parameters in EEG. EEG pattern for left, right and forward intentions will be analysed by researchers to discriminate among these different movement imagination-related EEG patterns [16, 17]. A paralysed person could communicate to a wheelchair by composing sequence of these mental stages, which will allow him/her to move left, right and forward.

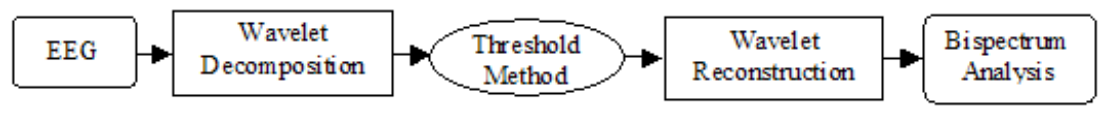

Figure 1: Flow of the algorithm. 


\subsection{Wavelet denoising technique}

Wavelets commonly used for denoising biomedical signals $[18,19]$ include the Daubechies 'db2', 'db8' and 'db6' wavelets and orthogonal Meyer wavelet. The wavelets are generally chosen whose shapes are similar to those of the normal EEG signal. The proposed wavelet denoising method is given as followed:

- Discrete Wavelet Transform: Use wavelet 'db6' with 4 levels of decomposition.

- Threshold: Estimate noise, select threshold, perform hard threshold.

- Inverse Discrete Wavelet Transform: Reverse process of decomposition.

\subsection{Bispectrum analysis}

The bispectrum or third order spectrum (HOS) has the advantage of suppressing Gaussian noise from EEG signal. Signal processing based on the first and second order moments and cumulants (SOS) and their spectral do not provide the full information of the acquired EEG signal. HOS carries both the magnitude and phase information, which will be used to recover the system impulse function and input impulse sequence from the linear time-invariant (LTI) system output signal. So the main advantage of HOS is that it can suppress Gaussian noise in detection, parameter estimation and solve classification problems, which is not possible by SOS. EEG signal is random by nature, so HOS method can be used for analysing the EEG signal due to its unique properties to random time series. As a result, there will be no contribution of system noise at the output bispectrum as the bispectrum of an additive Gaussian noise white signal is identically zero. The approach to system reconstruction will begin from the bispectrum of a LTI system output signal. The frequency domain system will be computed from the frequency domain information. The output bispectrum will provide the systems magnitude and phase information if the system input signal is non-Gaussian, while the same bispectrum will be zero if the system is driven by a Gaussian input signal. If a non-Gaussian stationary random process is applied as an input to a linear system, the output bispectrum is the product of the skewness of the input signal and the bispectrum of the system. Therefore, the system impulse response can be reconstructed from the bispectrum of a LTI system output signal. Therefore, in this proposed research, a technique called Cepstrum of Bispectrum can be applied for system reconstruction.

\subsection{Hardware modelling}

The hardware description language, VHDL will be used to model the algorithm. Proper simulation will be carried out to test the functionality of the proposed system. This is followed by the circuit synthesis that performs the translations of hardware description language code into an equivalent netlist of digital cells. The synthesis helps to integrate the design work and provides a higher feasibility to 
explore a far wider range of architectural alternative. Finally the code will be downloaded into the FPGA chip. The simulation and synthesis process will be carried out using Altera quartus software.

Based on the principle of functional evidence, it is demonstrated that EEG signal analysis and characterization can be carried out using HOS after applying wavelet denoising technique. The new FPGA implementation (Stratix II) of HOS algorithm will reduce high computational burden, which will run in real-time providing faster computational speed, higher performance at a lower cost.

\subsection{Hardware interface with wheelchair}

After the successful hardware prototyping, it will be interfaced with the power wheelchair. Power wheelchair has joysticks to control the chair as means of navigation. The proposed hardware will replace the existing control device so that the hardware module can perform the necessary navigation through brain signals based on the individual's intention. Figure 2 shows the architecture of the entire intelligent wheelchair system. Hardware module will generate decisions (left/right/forward movement) based on the EEG patterns after the signal processing depending on the individual's intention.

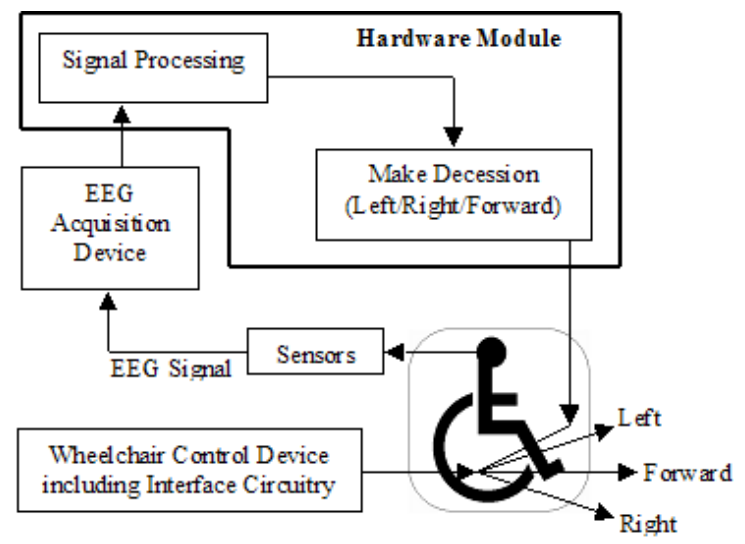

Figure 2: Architecture of the intelligent wheelchair system.

\section{Conclusion}

Wavelet provides better and powerful complement to conventional noise removal techniques like notch filters and frequency domain filtering methods. On the other hand HOS is also preferable because it can suppress Gaussian noise. Thus, this research proposes to employ both techniques to have a better and efficient processing of EEG signal, which can successfully filter recording noise and remove Gaussian noise for analysis and characterization to develop the intelligent wheelchair. 
VHDL will be used to model the algorithm, which will be followed by extensive testing and simulation to verify the functionality of the algorithm that allows efficient FPGA implementation. The chip will be cost effective, portable and robust for human computer interfacing devices like EMG related biomedical equipments, a wheelchair.

When fully developed, such a system will be a very useful device (wheelchair) especially for the severe disabled people.

\section{Acknowledgement}

The authors would like to express sincere gratitude to the Ministry of Higher Education for providing Fundamental Research Grant Scheme (FRGS) fund for the research (FRGS0106-21).

\section{References}

[1] Nakanishi S., Kuno Y., Shimada N., and Shirai Y., "Robotic Wheelchair Based on Observations of Both User and Environment," in Proc. of IROS'99, pp. 912-917, 1999.

[2] Felzer T., Freisleben B., "HaWCoS: The "hands-free" wheelchair control system," Proceedings of the Fifth International ACM SIGCAPH Conference on Assistive Technologies..: ACM, 2002, pp. 127-134. Edinburgh, Scotland, U.K

[3] Moon I., Lee M., Ryu J., and Mun M., "Intelligent Robotic Wheelchair with EMG-, Gesture-, and Voice-based Interfaces," Proc. of IEEE/RSJ IROS 2003, 2003.

[4] Mason S. G., Birch G. E., “A Brain-Controlled Switch for Asynchronous Control Applications”, IEEE Trans. Biomed. Eng., vol. 47, no. 10, October 2000

[5] Birch G. E., Lawrence P. D., and Hare R. D., "Single trial processing of event related potentials using Outlier information," IEEE Trans. Biomed. Eng., vol. 40, no. 1, pp. 59-73, Jan 1993.

[6] Lisha S., Minfen S, "Analysis of Non-stationary Electroencephalogram Using the Wavelet Transform", Proceedings of the $6^{\text {th }}$ International Conference on Signal Processing, Vol. 2, pp 1520-1523, 2002.

[7] Ramanan S.V., Kalpakam N.V., Sahambi J.S., "A novel wavelet based technique for detection and de-noising of ocular artifact in normal and epileptic electroencephalogram", International Conference on Communications, Circuits and Signals (ICCCAS 2004), Vol. 2, pp 27-29, 2004.

[8] Mallat S., "Multiresolution Frequency Channel Decomposition of Images and Wavelet Models", IEEE Transactions on ASSP, Vol. 37, No. 12, pp 2091-2110, 1989.

[9] Shuren Q., Zhong J., "Multi-Resolution Time-Frequency Analysis for Detection of Rhythms of EEG Signals", $3^{\text {rd }}$ IEEE Signal Processing 
Education Workshop and $11^{\text {th }}$ IEEE Digital Signal Processing Workshop 2004, pp. 338-341, 2004.

[10] Yamaguchi C., "Fourier and Wavelet Analyses of Normal and Epileptic Electroencephalogram (EEG)", Proceedings of $1^{\text {st }}$ International IEEE EMBS Conference on Neural Engineering, pp. 406-409, 2003.

[11] Yong L., Shengxun Z., "Apply Wavelet Transform to analyse EEG Signal", Proceedings of the $18^{\text {th }}$ Annual International Conference of the IEEE Engineering in Medicine and Biology Society, Col. 3, pp. 10071008, 1996.

[12] Rose D.A., Benjamin I., Clark I., Biscay R., Echeverria M., Virues T., "Multiresolution Decomposition of Non-Stationary EEG signals: A Preliminary Study", Computers in Biology and Medicine, Vol. 25, No. 4, pp. 373-382, 1995.

[13] Muthuswamy J., Sherman D. L., Thakor N. V., "Higher-Order Spectral Analysis of Burst Patterns in EEG", IEEE Transactions on Biomedical Engineering, Vol. 46, no. 1, pp. 92-99, 1999.

[14] Pomfrett C. J. D., Pearson A. J., "EEG Monitoring using Bispectral Analysis", IEE Colloquium on New Measurements and Techniques in Intensive Care, Digest no. 1996/179, pp. 5/1-5/3, 1996.

[15] C. E. Cummings, "Verilog simulation Xilinx designs", Proceeding of the International Verilog HDL Conference, Santa Clara, CA, pp. 93-100, 1994.

[16] Schloegl A., Neuper C., Pfurtscheller G., "Subject Specific EEG Patterns During Motor Imaginary", Proceedings - 19th International Conference IEEE/EMBS Oct. 30 - Nov. 2, 1997 Chicago, IL. USA

[17] Leeb R., Pfurtscheller G., "Walking through a Virtual City by Thought", Proceedings of the 26th Annual International Conference of the IEEE EMBS, September 1-5, 2004 San Francisco, CA, USA

[18] P. Carre, H. Leman, C. Fernandez, C. Marque, "Denoising of the uterine EHG by an undecimated wavelet transform, IEEE Trans. on Biomedical Signal Processing, vol 45, issue 9, pp 1104-1114, 1998

[19] S. Mallat, "A wavelet tour of signal processing", $2^{\text {nd }}$ edition, Academic Press, 1998, New York, USA 